\title{
Evaluation of a Pharmacist-Based Intervention to Reduce Readmissions in Geriatric High- Utilizer Patients: A Pilot Study
}

\author{
Sara Turbow, MD, $\mathrm{MPH}^{1}$; Kruti Shah, PharmD ${ }^{2}$; Katherine Penziner, $\mathrm{MD}^{3}$; Michael Knauss, PharmD ${ }^{2}$ \\ ${ }^{1}$ Division of General Medicine and Geriatrics, Department of Medicine, Emory University School of Medicine, Atlanta, GA \\ ${ }^{2}$ Department of Pharmacy Services, Grady Health System, Atlanta, GA \\ ${ }^{3}$ Yale University School of Medicine, New Haven, CT
}

\begin{abstract}
Purpose: The goal of this study was to determine if a pharmacist-led intervention to improve medication safety at hospital discharge reduced the number of hospital readmissions among geriatric high-utilizer patients. This study is the first to test a pharmacist-based intervention in a high-utilizer population.

Methods: This was a quasi-experimental pilot study done at a safety-net hospital in the southeastern US. Fifty-seven patients 65 years old and older who were in the $95^{\text {th }}$ percentile for number of hospital admissions in a year were included. On the day of discharge, one of the study pharmacists reviewed the discharge medication list and calculated the Medication Appropriateness Index (MAl) for each medication and reviewed for Beers Criteria. Any medication identified as potentially high-risk or inappropriate was flagged by the pharmacist and discussed with the team. The primary outcome was the number of admissions in the year following the intervention in the intervention group versus the control group.

Results: There were no statistically significant differences in the number of admissions, the MAl scores, or the number of medications meeting Beers Criteria between the two groups.

Conclusion: Although this study did not demonstrate a decrease in hospital admissions, it shows that pharmacist review of medications at discharge can identify potentially unnecessary medications that could lead to confusion or adverse events. Further research is necessary to identify interventions to prevent readmissions in this high-risk population.
\end{abstract}

Keywords: high-utilizers, readmissions, polypharmacy, medication appropriateness index, Beers Criteria, geriatrics

\section{Introduction}

In 2015 , health care accounted for $17.8 \%$ of the U.S.' $\$ 18.5$ trillion economy(1), and in the next eight years it is expected to rise to nearly $20 \%(2)$. Strikingly, $50 \%$ of health care costs are generated by only $5 \%$ of patients $(2,3)$. Though the population of high utilizers is very heterogeneous, they are more likely to be older, less educated, and publicly insured(4-6). Many have markedly worse outcomes than their peers, despite receiving more health care(6-8). These patients are of great interest to stakeholders at all levels, including physicians, hospital administrators, public (Medicare, Medicaid) and private payers, and policy makers.

Polypharmacy, while an important marker of health and intensity of medical care, does not tell the whole story. Highrisk medications are a known contributor to adverse events and short-term ( $\leq 30$-day) readmissions in elderly patients(9). However, it is not known how much they contribute to readmission risk beyond thirty days. While long-term readmissions are not penalized by insurance companies the same way short-term readmissions are, they are detrimental to patients and place a similar burden on the healthcare system.

Corresponding author: Sara Turbow, MD, MPH

Division of General Medicine and Geriatrics

Department of Medicine, Emory University School of Medicine 49 Jesse Hill Jr Dr SE, Atlanta, GA 30303

Phone: 404-251-8897; Email: sara.turbow@emory.edu
Pharmacist-driven interventions designed to reduce the use of high-risk medications have been developed to reduce medication-related adverse events(10) and hospital readmissions $(11,12)$ among geriatric patients. A recent study completed at Vanderbilt University examined the effect of pharmacist counseling in patients with acute coronary syndrome and decompensated heart failure on emergency department use and 30-day readmission rates and found a beneficial effect of the intervention in patients with low health literacy scores(13). Pharmacists, as key members of an interprofessional health care team, are well positioned to have a positive impact on medication and polypharmacy.

High utilizers, because of frequent readmissions, fragmented care, and negative social determinants of health, are at risk for complex medication regimens that may include high-risk or unsafe medications. The oldest patients in this population are at even greater risk, as they may suffer from unexpected side effects of medications or have additional difficulty managing a complex regimen. Here, we describe a pilot study evaluating the impact of a pharmacist-based intervention to improve medication safety at hospital discharge on readmission rates among geriatric high-utilizer patients.

\section{Methods \\ Study Design}

This is a quasi-experimental pilot study of a pharmacist-based intervention to improve medication safety and reduce hospital 
readmissions in a population of geriatric patients with a history of frequent admissions to the hospital.

\section{Patient Population}

Patients were eligible for inclusion in the study if they were 65 years old or older, had been admitted to Grady Health System (GHS) three or more times in 2015 or 2016, and were currently admitted to an internal medicine service at GHS. Patients were not eligible if they did not speak English, were incarcerated, were unable to consent for the study due to illness or dementia and did not have family or a medical decision maker available, or if they were planned to discharge from the hospital to a nursing home or hospice. Additionally, patients were not eligible if they were admitted to GHS's Acute Care of the Elderly (ACE) unit during the hospitalization in which they would be enrolled in the study. This is a multidisciplinary team focused on preventing decline of geriatric patients while hospitalized and provides a comprehensive post-discharge follow up plan(14). The ACE unit only had ten beds at the time of this research, so a fraction of all admitted elderly patients were not eligible for this reason.

\section{Patient Identification and Enrollment}

Patients were identified by reviewing a list of all patients admitted to internal medicine teams during the prior day. If on chart review a patient met inclusion criteria and there were no exclusion criteria identified, the medical team was notified of the patient's appropriateness for inclusion. The patient was then approached for enrollment by a study team member. Verbal and written consent was obtained from the patient or their medical decision maker. All study procedures and consents were approved by the Emory University Institutional Review Board and the Grady Health System Research Oversight Committee.

Baseline data was obtained from the participant's electronic medical record (EMR), Epic Systems (Verona, WI). This included demographics (name, medical record number, date of birth, sex), medical history (from progress notes including the history and physical and discharge summaries), past hospital admissions, and medications prescribed prior to hospital admission. The Charlson Comorbidity Index, a tool designed to measure the longitudinal risk of mortality based on comorbid conditions in longitudinal studies, was calculated based on patient's medical history $(15,16)$. All data was stored in RedCap(17).

\section{Medication Appropriateness Index \& Beers Criteria}

The Medication Appropriateness Index (MAI) is a 10-question tool developed to assess the level of inappropriateness of a medication(18,19). The score ranges from $0-18$, with 0 representing an appropriate medication and 18 representing a maximally inappropriate medication. It has been used in a number of different research settings and has good inter-rater reliability between pharmacists and physicians(10). The MAI questions are listed in the supplemental material (Table S1).
The Beers Criteria is a list of medications deemed potentially inappropriate in elderly patients that is published by the American Geriatrics Society(20). At the time of this study, the Beers Criteria was most recently updated in 2015.

\section{Intervention}

On the day of discharge, an email was sent to the medical team to remind them to contact one of the study pharmacists once the discharge medication reconciliation had been completed. Once the pharmacist was notified, they reviewed the discharge medication list and calculated the MAI for each medication and reviewed for Beers Criteria. Any medication identified as potentially high-risk or inappropriate, based on their MAl score or Beers Criteria, was flagged by the pharmacist and shared with the team. Recommendations were made to the primary team by the pharmacist but no decisions about stopping or changing medications were made by the research team. The pharmacists reviewed the EMR after discharge to record which medications, if any, were changed.

\section{Outcomes}

The primary outcome of this study was the number of hospital readmissions in the year following the intervention compared to the number of admissions they had in the calendar year prior to enrollment (2015 or 2016). Secondary outcomes included the effect of the pharmacy intervention on the overall use of high-risk medications in this population, and the change over the following year on the use of high-risk medications as defined by their MAl score and presence of Beers Criteria medications.

\section{Control Group}

Patients who were discharged without receiving the intervention were placed in the control group. They may not have received the intervention because the medical team forgot to alert the study team when the patient was leaving or the patient was discharged in the evening or on a weekend when the study team was not available. The patients who do not receive the intervention only had the baseline chart review and final follow-up data collected one year after the hospital admission during which they were enrolled.

\section{Intervention Group}

Participants who received the intervention were contacted via telephone at one month, three months, and six months after hospital discharge. A study team member contacted the participant and obtained a current medication list based on participant self-report. If the study team member was unable to reach the participant, two more attempts were made over the following one to two weeks before the follow-up encounter was closed. This duration was chosen so that the follow-up data collection occurred as close as possible to the intervals in the study while still giving the research team a variety of times and days of the week to contact the participant. 
The medical charts of all study participants were reviewed one year after the hospital admission at which they were enrolled. Data collected from this chart review included current medical diagnoses, hospital admissions since enrollment in the study, current medications, and if the patient had died in the year since enrollment.

\section{Data Analysis}

All data analysis was performed in SAS 9.2 (Cary, NC). Medications were categorized into the following groups: neurologic, cardiac, gastrointestinal, endocrine, pulmonary, mental health/pain, renal/genitourinary, infectious diseases, and other. Chi-squared tests were performed on categorical variables, and t-tests were performed on continuous variables. One-way ANOVA was used to compare the number of admissions in the year prior to enrollment and the year after enrollment in the intervention and control groups for patients who were high-utilizers in 2015 and 2016; one-way ANOVA with a post-hoc Tukey test was used to compare number of medications and average MAl across time points. P was set at 0.05 .

\section{Results}

A total of 57 patients were included in the study. Baseline demographics are shown in Table 1. Among patients who were high-utilizers in 2015, patients in the intervention group had more admissions at baseline than those who were not in the intervention group. Patients who did not receive the intervention were more likely to have died in the year following the intervention than those who received the intervention. Notably, there was no statistically significant difference in the Charlson Comorbidity Index $(\mathrm{CCl})$ between the two groups, although there was a trend towards the "no intervention" group having a higher $\mathrm{CCl}(\mathrm{p}=0.079)$.

Table 2 illustrates the change over time among patients in the intervention and no intervention groups. Figures 1 and 2 illustrate that there was no difference in the primary outcome of number of admissions in the year following enrollment between the two groups.

Table 3 lists the medications that were flagged by the pharmacist at discharge. A total of fourteen medications (5.9\%) were flagged. The most common reason that the medication was flagged was that it was deemed not indicated by the pharmacist. Of the flagged medications, approximately $60 \%$ were changed by the medical team. The most common change made was for the medication to be discontinued.

The average medication appropriateness index score and percent of Beers Criteria medications at the enrollment admission, discharge, and at the final follow-up one year after enrollment for all medications combined as well as each category of medications is listed in the supplemental material (Table S2), along with a list of the medications in each category (Table S3). Gastrointestinal medications had the highest average MAl score and the most Beers Criteria medications. Mental health and pain medications had significantly more Beers Criteria medications on admission among patients who did not receive the intervention, and their higher average MAI score trended towards significance. This difference became less pronounced at discharge and at the final follow-up.

\section{Discussion}

This study focused on a group of patients at risk for complications from high-risk medications: elderly patients in the 95th percentile for most admissions in a year at our hospital. While we did not see a statistically significant difference in the rate of hospital readmission at one year between the intervention and no intervention groups, there are still some important observations from this pilot study.

The pharmacist intervention did not have a statistically significant impact on the average MAl of medications prescribed-in the intervention group the MAl on admission was 0.76 and at discharge was $0.58(p=0.394)$; the control group's MAl was also unchanged between admission and discharge (0.95 vs. $0.91, p=0.853)$. The medications that were flagged by the pharmacist at discharge and eventually discontinued were primarily medications that were not indicated-for example, vitamins without documented vitamin deficiencies. While these medications are not "high risk," we argue that each additional medication in a regimen adds complexity and possibly confusion for patients like these who are often taking more than 10 medications. For example, the primary driver of increased MAI scores and frequency of Beers Criteria medications in the gastrointestinal medications group were proton pump inhibitors (PPI). It is unlikely that stopping these medications would prevent a readmission but removing them may decrease confusion and prevent short- and longterm side effects(10).

Interestingly, no medication received the highest MAI score of 18. The highest score given was a medication that had been prescribed prior to admission in a patient with clear contraindications to the medication. Among medications prescribed during the study period, the category with the highest average MAl score was gastrointestinal medications. This was primarily driven by PPIs being prescribed without a stop date.

Overall, the data indicate the medical teams were attentive to medication safety in this population, with only 14 medications identified by the pharmacists as inappropriate in the control group. Additionally, $35.4 \%$ of the medications in the pain and mental health category on the medication lists of patients in the control group on admission were on the Beers Criteria; this decreased to $27.9 \%$ at discharge without pharmacist intervention. However, this attention to the medication list could have been an unintended consequence of informing the medical teams that their patients were enrolled in the study. 
Focusing on medication appropriateness instead of polypharmacy sets our approach apart. Many of our patients have significant medical problems that necessitate multiple medications. These medications may be required; only trying to reduce the number of medications the patient receives is not always the best approach. For patients in the intervention group, over time the average number of medications increased from 9.8 to 11.8 but average MAl decreased from 0.76 to 0.61 , indicating that the patient population may be getting sicker but the medication use is appropriate. We hope that by making their overall medication list safer and more appropriate that they will only be on the medications that are absolutely necessary.

There are several limitations of this study. Sample size is a major limitation and may be the reason we don't have more significant results. Additionally, patients who were discharged on the weekends or in the evening did not receive the intervention because the pharmacist was not available at these times. There may be concern that these patients were less sick (hence why the medical team felt comfortable discharging them at these times), however, the $\mathrm{CCl}$ of patients in the control group was not statistically significantly different than the $\mathrm{CCl}$ of the intervention group, indicating that their baseline health was not that different. Finally, the use of patient self-report for the medication history at the various time points may have skewed the results at the one, three, and six-month phone calls, as patients may not be able to remember all of the medications they were prescribed.

The problem of high-utilization is extremely complex, and no single intervention will be able to solve it. This project represents an initial step into developing targeted interventions for this population. This can be a challenging population to work with because of their multiple medical problems and overlying social issues. While inpatient interventions can be complicated, this may be a model that is effective for this population. The successes and challenges we encountered in this study will inform the development of future interventions.
Ethics Approval: The study was approved by the Emory Institutional Review Board and the Grady Health System Research Oversight Committee. All patients provided written and verbal consent prior to participating.

Consent for Publication: Not applicable

Availability of Data and Materials: The data generated during the current study are not publicly available due to patient confidentiality, but may be available from the corresponding author on reasonable request.

\section{Key Points}

1. Geriatric high-utilizers, because of frequent readmissions and fragmented care, are at risk for complex medication regimens that may include unsafe medications.

2. This quasi-experimental pilot study of a pharmacistbased intervention to improve medication safety and reduce hospital readmissions in a population of geriatric patients with a history of frequent admissions to the hospital did not show a difference in the rate of hospital readmissions between the two groups.

3. The intervention did improve the average Medication Appropriateness Index score of patients at discharge.

\section{References}

1. Bodenheimer T. Strategies to Reduce Costs and Improve Care for High-Utilizing Medicaid Patients: Reflections on Pioneering Programs. Center for Health Care Strategies; 2013 p. 23.

2. Jiang $H J$, Weiss $A$, Barrett $M$, Sheng $M$. Characteristics of Hospital Stays for Super-Utilizers by Payer, 2012. HCUP Statistical Brief \#190 Rockville, MD; 2015.

3. Mitchell E. and Machlin S. Concentration of Health Expenditures and Selected Characteristics of High Spenders, U.S. Civilian Noninstitutionalized Population, 2015. Statistical Brief \#506. December 2017. Agency for Healthcare Research and Quality, Rockville, MD. http://www.meps.ahrq.gov/mepsweb/data_files/pub lications/st506/stat506.pdf

4. Hasselman D. Super-Utilizer Summit: Common Themes from Innovative Complex Care Management Program. Robert Wood Johnson Foundation; 2013 p. 32.

5. Lopez-Gonzalez L, Pickens GT, Washington R, Weiss AJ. Characteristics of Medicaid and Uninsured Hospitalizations, 2012. Rockville, MD: Agency for Healthcare Research and Quality; 2014 Oct pp. 1-15.

6. Steiner CA, Friedman B. Hospital utilization, costs, and mortality for adults with multiple chronic conditions, Nationwide Inpatient Sample, 2009. Prev Chronic Dis. 2013 Apr 25;10:E62. 
7. Moe J, Kirkland S, Ospina MB, Campbell S, et al. Mortality, admission rates and outpatient use among frequent users of emergency departments: a systematic review. Emerg Med J. 2016 Feb 18;33(3):230-6.

8. Nighswander TS. High utilizers of ambulatory care services: 6-year followup at Alaska Native Medical Center. Public Health Rep. 1984 Jul-Aug;99(4):400-4.

9. Pavon JM, Zhao Y, McConnell E, Hastings SN. Identifying risk of readmission in hospitalized elderly adults through inpatient medication exposure. J Am Geriatr Soc. 2014 ed. 2014 Jun;62(6):1116-21.

10. Spinewine A, Swine C, Dhillon S, et al. Effect of a Collaborative Approach on the Quality of Prescribing for Geriatric Inpatients: A Randomized, Controlled Trial. J Am Geriatr Soc. 2007 Apr 12;55(5):658-65.

11. Christensen $M$, Lundh A. Medication review in hospitalised patients to reduce morbidity and mortality. Cochrane Database Syst Rev. 2016 ed. 2016 Feb 20;2:CD008986.

12. Dedhia P, Kravet S, Bulger J, et al. A Quality Improvement Intervention to Facilitate the Transition of Older Adults from Three Hospitals Back to Their Homes. J Am Geriatr Soc. 2009 Aug 17;57(9):1540-6.

13. Bell SP, Schnipper JL, Goggins K, et al. Effect of Pharmacist Counseling Intervention on Health Care Utilization Following Hospital Discharge: A Randomized Control Trial. J GEN INTERN MED. 2016 May;31(5):470-7.

14. Fox MT, Persaud M, Maimets I, et al. Effectiveness of Acute Geriatric Unit Care Using Acute Care for Elders Components: A Systematic Review and MetaAnalysis. J Am Geriatr Soc. 2012 Nov 23;60(12):223745.
15. Charlson M, Wells MT, Ullman R, et al. The Charlson Comorbidity Index Can Be Used Prospectively to Identify Patients Who Will Incur High Future Costs. Catapano A, editor. PLoS ONE. 2014 Dec 3;9(12):e112479-16.

16. Frenkel WJ, Jongerius EJ, Mandjes-van Uitert MJ, et al. Validation of the Charlson Comorbidity Index in Acutely Hospitalized Elderly Adults: A Prospective Cohort Study. J Am Geriatr Soc. Wiley/Blackwell (10.1111); 2014 Jan 21;62(2):342-6.

17. Harris PA, Taylor R, Thielke R, et al. Research electronic data capture (REDCap)--a metadata-driven methodology and workflow process for providing translational research informatics support. J Biomed Inform. 2008 ed. 2009 Apr;42(2):377-81.

18. Samsa G, Hanlon JT, Schmader KE, et al. A Summated Score for the Medication Appropriateness Index: Development and Assessment of Clinimetric Properties Including Content Validity. J Clin Epidemiol. 1994;47(8):891-6.

19. Hanlon JT, Schmader KE. The Medication Appropriateness Index at 20: Where It Started, Where It Has Been, and Where It May Be Going. Drugs Aging. 2nd ed. 2013 Sep 24;30(11):893-900.

20. The American Geriatrics Society 2015 Beers Criteria Update Expert Panel. American Geriatrics Society 2015 Updated Beers Criteria for Potentially Inappropriate Medication Use in Older Adults. J Am Geriatr Soc. 2015 Oct 8;63(11):2227-46. 
Table 1: Demographics

\begin{tabular}{|l|l|l|l|}
\hline & \multicolumn{1}{|c|}{ Intervention (n=21) } & \multicolumn{1}{|c|}{ No Intervention (n=36) } & \multicolumn{1}{c|}{ p } \\
\hline \% Men & $42.8 \%(9)$ & $50 \%(18)$ & 0.602 \\
\hline Mean Age (range) & $70.9(66-86)$ & $72.8(65-86)$ & 0.272 \\
\hline $\begin{array}{l}\text { Number of Admissions during year of high- } \\
\text { utilization }\end{array}$ & $2015: 5.3(3-13)$ & $2015: 3.8(3-6)$ & $0.045^{*}$ \\
\hline Average LOS (range) & $2016: 4.3(3-9)$ & $2016: 3.7(3-6)$ & 0.258 \\
\hline Charlson Comorbidity Index Mean (range) & $2015: 3.9(1.5-9.6)$ & $2015: 3.8(2.0-10.3)$ & 0.856 \\
\hline \% Discharged Home & $2016: 3.8(2.0-7.2)$ & $2016: 6.1(2.0-17.3)$ & 0.124 \\
\hline $\begin{array}{l}\text { \% died of patients whose outcomes are } \\
\text { known }\end{array}$ & $85.7 \%(\mathrm{n}=18)$ & $4.2(1-9)$ & 0.079 \\
\hline
\end{tabular}

*significant at $\mathrm{p}=0.05$

Table 2: Change Over Time of Number of Medications and Average Medication Appropriateness Index

\begin{tabular}{|c|c|c|c|c|c|c|c|}
\hline & Baseline & Discharge & 1 Month & 3 Months & 6 Months & $\begin{array}{l}1 \text { Year } \\
\text { (Final) }\end{array}$ & $\mathbf{p}$ \\
\hline \multicolumn{8}{|c|}{ Intervention } \\
\hline Number of Patients & 21 & 21 & 13 & 7 & 10 & 20 & \\
\hline Average Number of Meds (range) & $9.8(0-18)$ & $11.5(4-18)$ & $11.2(4-16)$ & $9.0(6-13)$ & $10.1(2-19)$ & $11.8(7-19)$ & 0.452 \\
\hline Average MAI (score range) & $0.76(0-11)$ & $0.58(0-8)$ & $0.41(0-2)$ & $0.77(0-2)$ & $0.82(0-2)$ & $0.62(0-2)$ & 0.601 \\
\hline \multicolumn{8}{|l|}{ No Intervention } \\
\hline Number of Patients & 36 & 36 & & & & 27 & \\
\hline Average Number of Meds (range) & $12.0(5-24)$ & $12.3(2-25)$ & & & & $11.8(0-22)$ & 0.769 \\
\hline Average MAI (score range) & $0.95(0-16)$ & $0.92(0-5)$ & & & & $0.88(0-2)$ & 0.876 \\
\hline
\end{tabular}

Table 3: Medications Flagged by Pharmacists and Outcomes

\begin{tabular}{|l|l|l|}
\hline \multicolumn{1}{|c|}{ Medication Name } & Why was this medication flagged? & \multicolumn{1}{c|}{ What was the medication changed to? } \\
\hline Albuterol & Duplicate & NA-no change \\
\hline Albuterol & Duplicate & NA-no change \\
\hline Albuterol-Ipratropium & Ineffective & Changed to alternate inhalers \\
\hline Atorvastatin & Cost & Changed to alternate statin \\
\hline Cyanocobalamin & Not indicated & Discontinued \\
\hline Dicyclomine & Not indicated & Discontinued \\
\hline Doxazosin & Not indicated & NA-no change \\
\hline Esomeprazole & Duration & NA-no change \\
\hline Esomeprazole & Inappropriate duration & NA-no change \\
\hline Folic Acid & Not Indicated & NA-no change \\
\hline Metoclopramide & Not indicated & Discontinued \\
\hline Ranitidine & Duplicate & Discontinued \\
\hline Thiamine & Not indicated & Discontinued \\
\hline Trazodone & Inappropriate dose & Changed dose \\
\hline
\end{tabular}


Table S1: Medication Appropriateness Index Questions (18)

\begin{tabular}{|l|c|}
\hline Question & Score \\
\hline Is there an indication for the drug? & 3 \\
\hline Is the medication effective for the condition? & 3 \\
\hline Is the dosage correct? & 2 \\
\hline Are the directions correct? & 2 \\
\hline Are the directions practical? & 2 \\
\hline Are there clinically significant drug-drug interactions? & 2 \\
\hline Are there clinically significant drug-disease/condition interactions? & 1 \\
\hline Is there unnecessary duplication with other drug(s)? & 1 \\
\hline Is the duration of therapy acceptable? & 1 \\
\hline Is this drug the least expensive alternative compared with others of equal utility? & 1 \\
\hline
\end{tabular}

